\title{
Functionally graded multi-material freeze-cast structures with continuous microchannels
}

\author{
Christiansen, Cathrine Deichmann; Nielsen, Kaspar Kirstein; Bjørk, Rasmus
}

Published in:

Journal of the European Ceramic Society

Link to article, DOI:

10.1016/j.jeurceramsoc.2019.12.018

Publication date:

2020

Document Version

Peer reviewed version

Link back to DTU Orbit

Citation (APA):

Christiansen, C. D., Nielsen, K. K., \& Bjørk, R. (2020). Functionally graded multi-material freeze-cast structures with continuous microchannels. Journal of the European Ceramic Society, 40(4), 1398-1406.

https://doi.org/10.1016/j.jeurceramsoc.2019.12.018

\section{General rights}

Copyright and moral rights for the publications made accessible in the public portal are retained by the authors and/or other copyright owners and it is a condition of accessing publications that users recognise and abide by the legal requirements associated with these rights.

- Users may download and print one copy of any publication from the public portal for the purpose of private study or research.

- You may not further distribute the material or use it for any profit-making activity or commercial gain

- You may freely distribute the URL identifying the publication in the public portal

If you believe that this document breaches copyright please contact us providing details, and we will remove access to the work immediately and investigate your claim 


\title{
Functionally graded multi-material freeze-cast structures with continuous microchannels
}

\author{
C. D. Christiansen, K. K. Nielsen, R. Bjørk*
}

\begin{abstract}
We present the processing of functionally graded multi-material freeze-cast structures, i.e. structures with varying material properties but continuous and homogeneous microchannels. We demonstrate this for stepwise, continuous, highly viscous and gelation freezing to achieve freeze-cast structures of graded $\mathrm{La}_{0.66} \mathrm{Ca}_{0.24} \mathrm{Sr}_{0.09^{-}}$ $\mathrm{Mn}_{1.05} \mathrm{O}_{3}$ (LCSM9) and $\mathrm{La}_{0.67} \mathrm{Ca}_{0.27} \mathrm{Sr}_{0.06} \mathrm{Mn}_{1.05} \mathrm{O}_{3}$ (LCSM6) ceramics.

The two phases are successfully distinguished from one another by the addition of $10 \mathrm{wt} \% \mathrm{Ce}_{0.9} \mathrm{Gd}_{0.1} \mathrm{O}_{2}$ (CGO) to the LCSM6 phase. The relative Ce-content facilitates tracking of the LCSM6 phase using energy-dispersive $X$-ray (EDS) elemental analysis. Coupled with scanning electron microscopy, we show well-defined interfaces with continuous microchannels between the two LCSM phases in both green and sintered samples.

By implementing constant freezing rates of $-1.5 \mathrm{~K} / \mathrm{min}$ a consistent freezing front velocity of $\sim 13 \mu \mathrm{m} / \mathrm{s}$ is maintained, and we found that a structural continuity can be maintained across the LCSM9-LCSM6/CGO interface, however, with varying pore morphology depending on the various freezing procedures.

Department of Energy Conversion and Storage, Technical University of Denmark - DTU, Anker Engelunds Vej 1, DK-2800 Kgs. Lyngby, Denmark

*Corresponding author: rabj@dtu.dk
\end{abstract}

\section{Introduction}

Through the past 20 years freeze-casting has become a versatile templating technique with a broad range of applications[1] within engineering of microchannels in polymer, metal and ceramic structures. However, thus far, ceramic freeze-casts consist of a single material or a composite of homogeneously distributed phases of materials. Freeze-casting of a functionally graded or layered structures with two or more phases of material separated by a distinct interface perpendicular to the channel orientation and continuous microchannels between the two phases has not yet been achieved. It would, however, be extremely useful in applications such as e.g. membraneand filter-applications, flow catalysis or magnetic refrigeration, as the material properties can thus be varied along the microchannels throughout the freeze-cast structure.

During ceramic freeze-casting, an aqueous suspension of ceramic particles is frozen directionally driven by an imposed thermal gradient. The growing ice crystals causes segregation of the particles, which results in a two-phase structure of ceramic and ice. Subsequently, the latter is removed by sublimation. Sintering of the freeze-cast green body results in a rigid, monolithic ceramic with directional porosity in the form of parallel, well-defined channels. The morphology and dimensions of these channels strongly depend on freezing conditions, while the porosity mainly depends on the ceramic load of the suspension $[2 ; 3 ; 4 ; 5 ; 6]$. The porosity is typically hierarchical with macroporosity in the form of larger, ice-templated pores - i.e. the channels - and inter-particle microporosity in the walls in the form of micropores.
The channels are directly shaped from the ice crystals and are in the ceramic green body the direct imprint of these. The continuity and low tortuosity of channels are due to the intrinsic properties of water during ice crystal formation under an applied thermal gradient. Layering of two separately frozen freeze-casts would therefore lead to a discontinuity in the channels across the interface between the two materials causing undesirable distortion of the otherwise homogeneous channels.

In order to achieve a structural continuity across the interface between two materials in freeze-casting, the freezing step should also be continuous across the interface ensuring continuous ice crystals. In order to do so, the materials must be layered in the wet stage, i.e. as liquid suspensions prior to freezing. However, upon being poured on top of each other with no further measures, the two suspensions will inevitably mix due to advection, convection and diffusion. An approach to inhibit this mixing and achieve a proper layering of liquid suspensions could be to alter the flow properties of the suspensions. In this work we investigate two approaches for doing so:

1. Inhibiting flow/miscibility by increasing viscosity using polymeric/steric additives

\section{Elimination of flow/miscibility by gelation}

Polyvinylpyrrolidone (PVP) has previously been studied for its effects on rheological properties of ceramic suspensions, where viscoelasticity and viscosity were found to depend on the average molecular weight and suspension concentration 
of PVP[7; 8]. Various PVP concentrations are thus added to ceramic suspensions for freeze-casting in this work to increase viscosity and thus inhibit flow and miscibility of suspensions in order to achieve a wet layering of these.

Gelation freeze-casting has successfully resulted in freezecast ceramics of well-defined parallel channels, however, with a slightly altered morphology $[9 ; 10 ; 6]$. Gelation freezecasting provide an additional processing step in the gelation of suspensions, where near net-shaping of the gelated suspension is possible if desired. If gelation is carried out such that the suspension looses fluidity, gelated suspensions can in principle be processed and stacked perpendicular to the freezing direction, resulting in a composite gelated sample of layered suspensions of different materials.

As a ceramic we use the perovskite $\mathrm{La}_{0.66} \mathrm{Ca}_{0.33-x} \mathrm{Sr}_{x^{-}}$ $\mathrm{Mn}_{1.05} \mathrm{O}_{3}$ (LCSM), which is well known for its desirable magnetocaloric properties. The Curie temperature of LCSM can be controlled by varying the strontium doping with achievable Curie temperatures in the range of $267 \mathrm{~K}$ to $375 \mathrm{~K}[11]$, making this material attractive for e.g. application as an active magnetic regenerator (AMR) material in magnetic refrigeration[12; 13]. Magnetic refrigeration utilizes the AMR cycle and offers an alternative to conventional refrigeration[14]. AMR technology is based on a porous magnetic material through which an environmentally friendly fluid flows in order to transfer heat. The efficiency of the AMR cycle strongly depends on the geometry of the regenerator material. In order to improve the efficiency an optimal structure could consist of microchannels with a large porosity while maintaining a large specific surface area[12]. Freeze-casting offers a processing route for structures meeting these specific criteria[15]. However, in order to achieve an optimal operational temperature span of such a regenerator, a chemically graded, or layered, structure is desired[13]. The magnetocaloric effect, i.e. the change in temperature of a ferromagnetic material upon varying an externally applied magnetic field, peaks around the Curie temperature of the sample. The Curie temperature, $T_{C}$, defines the transition temperature between the ferro- and paramagnetic states. Joining multiple similar materials with slightly varying chemical properties and thus varying $T_{C}$, enhances the temperature range over which the AMR device operates[13; 16]. Magnetic refrigeration is thus one possible application that would benefit greatly by graded freeze-cast structures.

In this work, graded freeze-cast structures of $\mathrm{La}_{0.66} \mathrm{Ca}_{0.24}$ $\mathrm{Sr}_{0.09} \mathrm{Mn}_{1.05} \mathrm{O}_{3}$ (LCSM9) and $\mathrm{La}_{0.67} \mathrm{Ca}_{0.27} \mathrm{Sr}_{0.06} \mathrm{Mn}_{1.05} \mathrm{O}_{3}$ (LCSM6) are engineered by optimizing the suspension viscosities with the addition of PVP of various concentrations and average molecular weights and by gelation-freezing and net-shaping using gelatine. As LCSM6 and LCSM9 are very similar chemically, a $\mathrm{Ce}_{0.9} \mathrm{Gd}_{0.1} \mathrm{O}_{2}$ (CGO) marker is added to the LCSM6 phase in order to distinguish the two phases chemically by energy-dispersive X-ray spectroscopy[17].

\section{Materials \& experimental procedures}

Graded freeze-cast ceramics were prepared from layered aqueous suspensions of LCSM powders. Samples were prepared from the same stock suspensions with various concentrations of either commercially available amino-based dispersant and binder, polymers such as polyvinylpyrrolidone (PVP) or gelatin in order to change suspension properties and freezing conditions to achieve a distinct layering of the final freeze-cast structure.

\subsection{Material properties and suspension preparation}

Two stock ceramic suspensions were prepared from 20 vol\% powders in MiliQ water: one suspension was prepared solely from $\mathrm{La}_{0.66} \mathrm{Ca}_{0.24} \mathrm{Sr}_{0.09} \mathrm{Mn}_{1.05} \mathrm{O}_{3}$ (LCSM9, CerPoTech), while the other was prepared from $\mathrm{La}_{0.67} \mathrm{Ca}_{0.27} \mathrm{Sr}_{0.06} \mathrm{Mn}_{1.05} \mathrm{O}_{3}$ (LCSM6, CerPoTech) and $\mathrm{Ce}_{0.9} \mathrm{Gd}_{0.1} \mathrm{O}_{2}(\mathrm{CGO}, \mathrm{CerPoTech})$ in weight ratio $9: 1$, in the following referred to as suspensions/samples LCSM9 and LCSM6/CGO, respectively. LCSM powders were calcined at $1000{ }^{\circ} \mathrm{C}$ for 2 hours prior to use. Material and suspension properties are summarized in Table 1.

To establish a sufficient dispersion of particles, $2.5 \mathrm{wt} \%$, solid to ceramic ratio, of dispersant (DURAMAX ${ }^{\mathrm{TM}}$ D-3005, Rohm and Haas, Dow Chemical) and dropvise addition of nitric acid to adjust the $\mathrm{pH}$ of suspensions from $\sim 8$ to $\sim 6.5$ was added. Suspensions were homogenized by ball milling in PE flasks using zirconia balls (Ø3-4 mm) as grinding media. To ensure even freezing kinetics and ice crystal growth through both layers of a sample, and thus homogeneous channel sizes, suspensions were ball milled until homogeneous and comparable in particle size distributions as shown on Figure 1. Thus, LCSM6/CGO was ball milled at $25 \mathrm{rpm}$ for $\sim 72$ hours while LCSM9 was ball milled at $45 \mathrm{rpm}$ for $\sim 72$ hours. Particle size distributions of milled suspensions were monitored using a Laser Diffraction Particle Size Analyser (LS 13 320, Beckman Coulter), while the density of calcined powders where measured by gas pycnometry (AccuPyc II 1340, Micromeritrics).

From these stock suspensions, suspensions of increased viscosity, section 2.1.1, suspensions for gelation, section 2.1.2, and samples for measurements of material characteristics were prepared.

Pellets for dilatometry and magnetization measurements were prepared by slip-casting given that the microporosity of

Table 1. Summarization of properties of LCSM6/CGO and LCSM9, where density was measured on powders that had been calcinated at $1000{ }^{\circ} \mathrm{C}$ for 2 hours, the median particle size, $\mathrm{d}_{50}$, is of suspensions after ball milling while onset temperature, $\mathrm{T}_{\text {onset }}$, and Curie temperature, $\mathrm{T}_{C}$, is measured on green and sintered pellets, respectively.

\begin{tabular}{ccccc}
\hline & $\begin{array}{c}\text { Density } \\
{\left[\mathrm{g} / \mathrm{cm}^{3}\right]}\end{array}$ & $\begin{array}{c}\mathrm{d}_{50} \\
{[\mu \mathrm{m}]}\end{array}$ & $\begin{array}{c}\mathrm{T}_{\text {onset }} \\
{\left[{ }^{\circ} \mathrm{C}\right]}\end{array}$ & $\begin{array}{c}\mathrm{T}_{C} \\
{[\mathrm{~K}]}\end{array}$ \\
\hline LCSM6/CGO & $6.10 / 6.92$ & 0.41 & 1015 & $288 /-$ \\
LCSM9 & 6.08 & 0.49 & 930 & 300 \\
\hline \hline
\end{tabular}




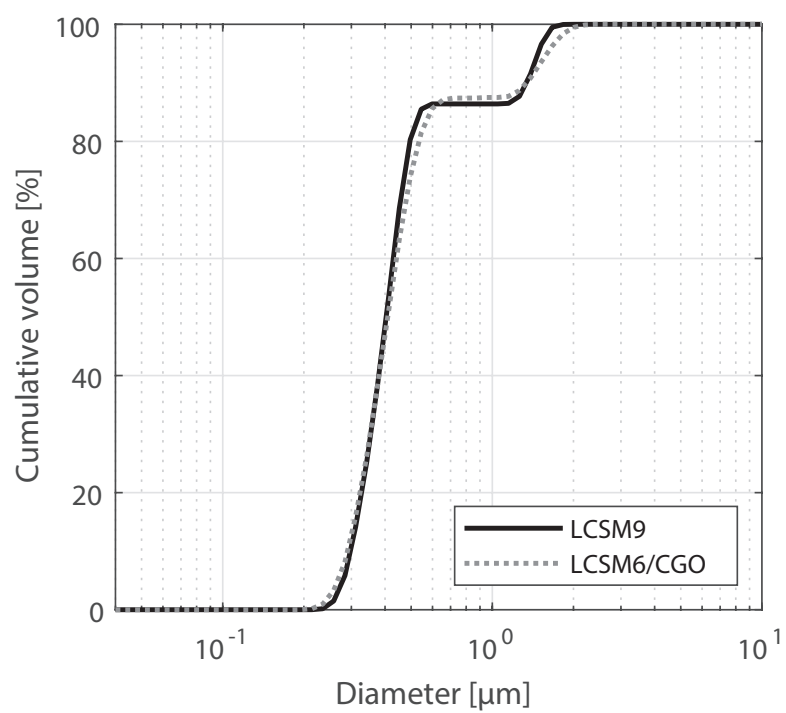

Figure 1. Particle size distribution of suspensions after ball milling reaching median particle sizes of $\mathrm{d}_{50}=0.49 \mu \mathrm{m}$ and $\mathrm{d}_{50}=0.41$ for LCSM9 and LCSM6/CGO suspensions, respectively.

slip-cast pellets to a larger degree resembles that of freezecasts compared to e.g. pressed pellets. For slip-casting, cylindrical polytetrafluoroethylene (PTFE) moulds with inner diameter of $6 \mathrm{~mm}$ were placed on an alabaster plaster medium. Stock suspensions for freeze-casting, with the addition of 2 wt $\%$ binder (DURAMAX ${ }^{\mathrm{TM}}$ B-1022, Rohm and Haas, Dow Chemical) was poured into the moulds and left to dry for at least 48 hours. Pellets for dilatometry were used in the green state while pellets for magnetization measurements were sintered using the same heating program as used for the freeze-cast samples (see section 2.2).

Dilatometry measurements were performed using a single push-rod dilatometer (DIL 402, NETZSCH) with a 2 hour hold at $550{ }^{\circ} \mathrm{C}$ (heating rate of $0.25 \mathrm{~K} / \mathrm{min}$ ) for burnout of additives and a 3 hour hold at $1100{ }^{\circ} \mathrm{C}$ (heating rate of 0.5 $\mathrm{K} / \mathrm{min}$ ) for densification under normal atmosphere (flow rate of $20 \mathrm{~mL} / \mathrm{min}$ ). Shrinkage curves were analyzed using Proteus Thermal Analysis software (NETZSCH) for evaluation of onset temperatures (see Supporting information, Figure S1) and summarized in Table 1.

Magnetization measurements for evaluation of the Curie temperature were obtained using a vibrating sample magnometer (VSM, 7407, Lake Shore Cryotonics). The isothermal magnetization was measured up to a maximum field of $\mu_{0} H_{\text {ext }}=1.5 \mathrm{~T}$ from $250 \mathrm{~K}$ to 340 with temperature steps of $2 \mathrm{~K}$ for determination of the Curie temperature and indirect measurements of the isothermal entropy change as shown in Figure 2.

\subsubsection{Viscosity}

Viscous suspensions were prepared from stock suspensions of LCSM6/CGO and LCSM9, respectively, by adding 5 or $10 \mathrm{wt} \%$, solid to ceramic ratio, polyvinylpyrrolidone K30

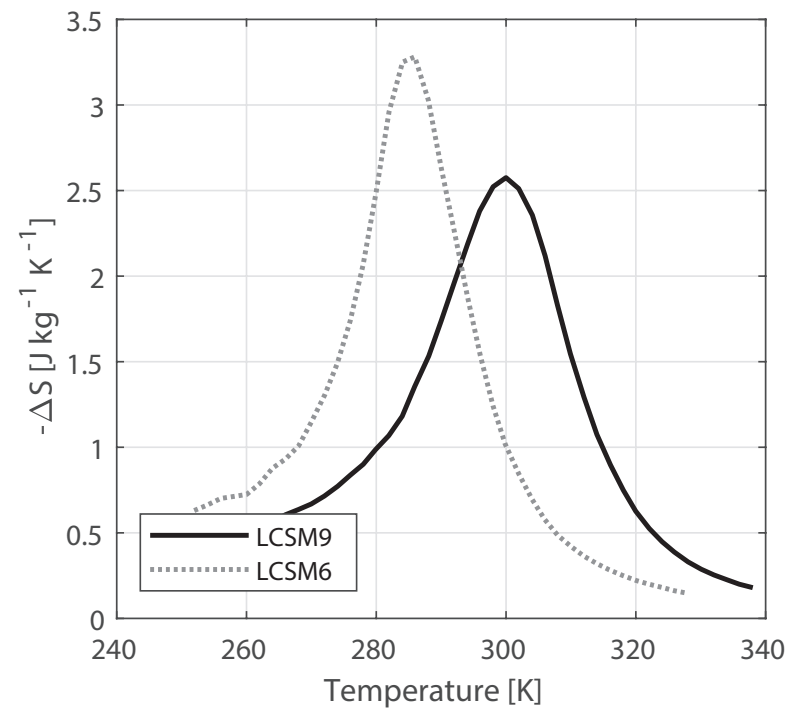

Figure 2. Isothermal entropy change for slip-cast, sintered pellets of LCSM6 and LCSM9 at an applied field of $1.5 \mathrm{~T}$, where the peak temperature can be estimated as the Curie temperature.

(PVP K30, $\mathrm{M}_{w} \sim 40,000$, Sigma-Aldrich) or $5 \mathrm{wt} \%$, solid to ceramic ratio, polyvinylpyrrolidone K90 (PVP K90, Fluka Chemika). For comparison, suspensions with the addition of $2 \mathrm{wt} \%$, solid to ceramic ratio, of a low viscosity commercial binder (DURAMAX ${ }^{\mathrm{TM}}$ B-1022, Rohm and Haas, Dow Chemical) were likewise prepared from both stock suspensions of LCSM6/CGO and LCSM9.

Suspensions were mixed on a low-energy ball mill at $10 \mathrm{rpm}$ for $\sim 24$ hours, after which viscosity curves of the eight suspensions were obtained using a rheometer (HAAKE Rheostress 600, Thermo Electron Corporation) equipped with a plate spindle (Platte P60 Ti L, Thermo Electron Corporation) with pre-shearing and reversing increase in shear rate from 0.1 to $50 \mathrm{~s} \mathrm{~s}^{-1}$ at $21^{\circ} \mathrm{C}$. The plate spindle was equipped with a solvent trap to minimize evaporation during measurements. Viscosity curves are shown in Figure 3, where it is evident that an increase in PVP load increases the viscosity.

Concentrations of PVP were chosen such that a sufficient range of viscosities was achieved. Increasing the PVP wt\% further was found to increase the viscosity further. However, increased concentrations of PVP in the suspensions lead to sticky and unmanageable suspensions that proved difficult to de-air and pour into moulds due to too large viscosity. Sintered freeze-cast ceramics would also often show severe crack formation. Thus, a maximum of $10 \mathrm{wt} \%$ PVP is used in the present work.

Viscous and standard suspensions were de-aired in vacuum and pre-cooled along with the polytetrafluoroethylene (PTFE) moulds in an ice bath for at least 30 minutes prior to freeze-casting to a temperature of approximately $0{ }^{\circ} \mathrm{C}$. This was done partly to ensure consistent freezing-conditions and partly to minimize the thermal energy in the system. 
(a)

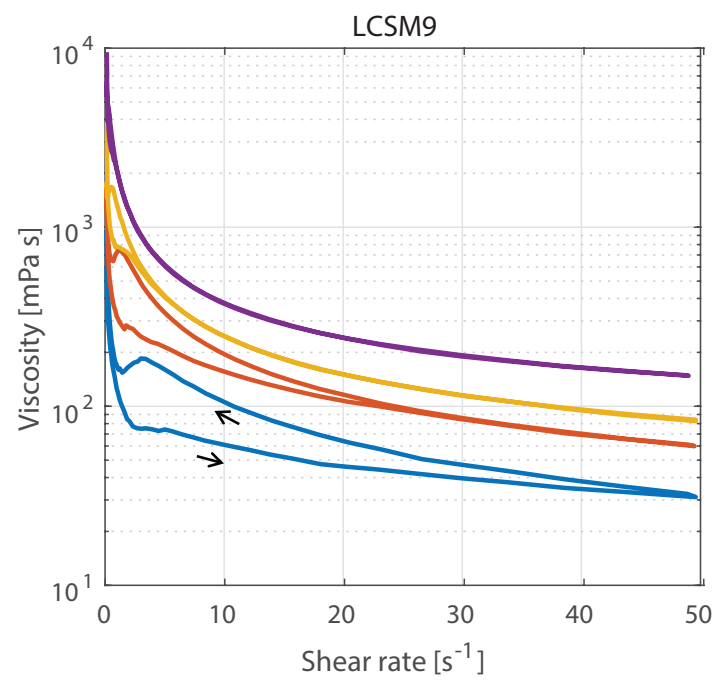

(b)

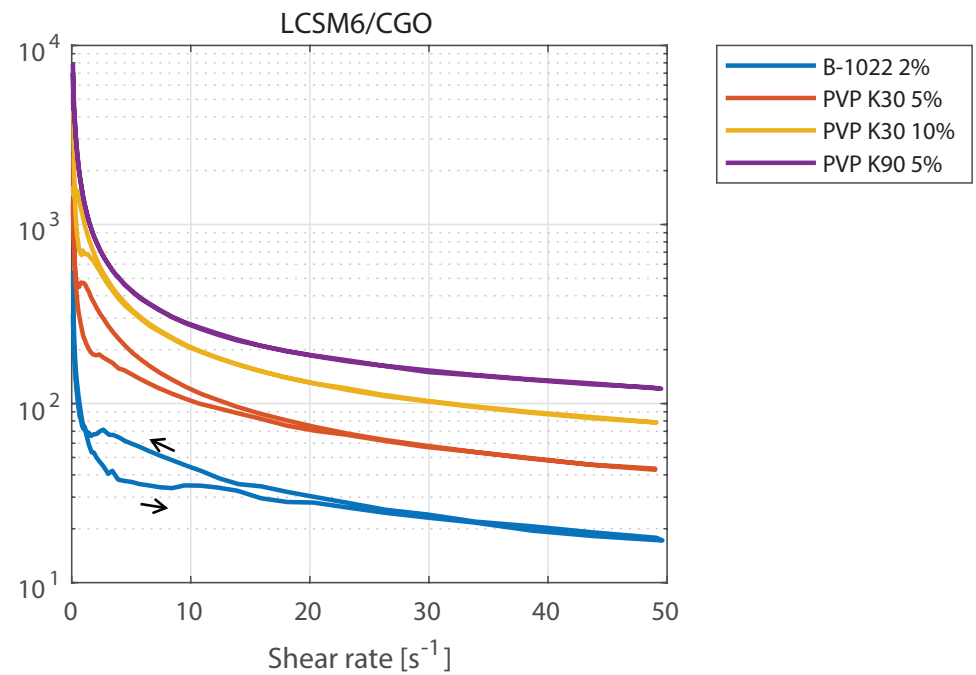

Figure 3. Viscosity curves of (a) LCSM9 and (b) LCSM6/CGO suspensions with 2.5\% DURAMAX ${ }^{\mathrm{TM}}$ B-1022 binder, 5 or 10 wt\% PVP K30 or $5 \mathrm{wt} \%$ PVP K90. The addition of the long polymers of PVP K30 and 90 significantly increases the viscosity of the suspensions. Arrows indicate the direction of measurements, and are only given for the bottom curve, but the trend is the same for all suspensions.

\subsubsection{Gelation}

Suspensions for gelation were prepared from stock suspensions of LCSM6/CGO and LCSM9, respectively, by adding $1.5 \mathrm{wt} \%$, solid to ceramic ratio, of gelatin (porcine skin, $\sim 175$ g Bloom, type A, Sigma-Aldrich). Gelatin was dissolved in suspensions by stirring at $45{ }^{\circ} \mathrm{C}$ for 30 minutes with a mechanical stirrer equipped with a propeller. The solutions were poured into pre-heated PTFE moulds $(\varnothing 20 \mathrm{~mm})$ equipped with copper bottoms and then immediately de-aired in a vacuumchamber for 5 minutes. Samples were left to harden at $2{ }^{\circ} \mathrm{C}$ for 17 hours.

Gelated suspensions were then carefully removed from the moulds and sliced into 1-2 cm cylindrical specimens. These were stacked such that each final sample were composed of a slice of LCSM6/CGO in the bottom and a slice of LCSM9 in the top. The composite samples were then carefully transferred back to a mould and left to settle at $2{ }^{\circ} \mathrm{C}$ for 1 hour before being frozen as described in section 2.2. The gelatin content was chosen such that the suspension just looses fluidity upon gelation and is manageable for cutting and stacking.

\subsection{Freeze-casting}

The freeze-casting set-up and general procedure is described in detail by Christiansen et al.[18]. A set-up with a thermoelectric element for precise temperature control of a copper coldfinger was used to directionally freeze samples. PTFE moulds ( $\varnothing 16$ or $20 \mathrm{~mm}$ ) equipped with thermocouples (Ktype, gauge 36 , Omega) along the height for monitoring of the freezing progression and a detachable copper bottom were used.

Generally, suspensions were frozen directionally in a PTFE mould by bringing the copper bottom of the mould into thermal contact with the coldfinger of the freeze-caster. Suspensions were frozen using a linear temperature profile, i.e. a constant decrease of the coldfinger temperature, of -1.5 $\mathrm{K} / \mathrm{min}$ from an equilibrated temperature of $275 \mathrm{~K}$. A linear temperature profile was chosen to ensure homogeneous channel sizes throughout the samples[3; 4; 5;6]. However, in order to achieve a distinct material interface, preparation of samples and freeze-casting procedure were varied depending on altered suspension or freezing conditions as follows:

Stepwise freezing was achieved by the initial freezing of one suspension, followed by freezing of the other suspension. Pre-cooled LCSM6/CGO suspension with B-1022 binder was poured into a mould open to ambient conditions of $\sim 5^{\circ} \mathrm{C}$. Sample and mould were equilibrated at a coldfinger temperature of $275 \mathrm{~K}$ for 300 seconds before being frozen using a constant temperature change of $-1.5 \mathrm{~K} / \mathrm{min}$. The suspension was frozen solid and the mould and frozen suspension were subsequently equilibrated at $270 \mathrm{~K}$ by adjusting the coldfinger temperature. Pre-cooled LCSM9 suspension was poured on top of the frozen suspension and frozen by immediately decreasing the temperature of the coldfinger from $270 \mathrm{~K}$ by $-1.5 \mathrm{~K} / \mathrm{min}$ until the top suspension was also completely frozen.

Continuous freezing follows the initial procedure of stepwise freezing, however, instead of letting the bottom suspension freeze solid, the LCSM9 suspension was added to the mould just before the freezing front, i.e. the solid/liquid interface, reached the top of the suspension. The freezing front position was evaluated by measuring the temperature along the height of the mould and the position of the freezing front was estimated at the position of $0{ }^{\circ} \mathrm{C}$. A constant temperature change rate of $-1.5 \mathrm{~K} / \mathrm{min}$ of the coldfinger was maintained during 
the entire freezing process until the sample was frozen solid.

Increased viscosity allowed a direct layering of the liquid suspensions. Composite samples were prepared from suspensions of LCSM6/CGO and LCSM9 of the same binder content, respectively. $3 \mathrm{~mL}$ of LCSM6/CGO suspension was poured into a mould. Carefully, another $3 \mathrm{~mL}$ of LCSM9 suspension was layered on top using a syringe equipped with a $1.2 \times 40 \mathrm{~mm}$ blunt filling needle. The tip of the needle was positioned such that it barely broke the surface of the first suspension at the approximate center of the mould and the other suspension was very slowly transferred to the mould in order to maintain a layering of suspensions. Mould and layered suspension were left open to ambient conditions of $\sim 5$ ${ }^{\circ} \mathrm{C}$ and equilibrated at a coldfinger temperature of 275 $\mathrm{K}$ for 300 seconds before applying constant temperature change rate of $-1.5 \mathrm{~K} / \mathrm{min}$ until the sample was frozen solid.

Gelation freezing was achieved by freezing the already gelated and stacked samples. Pre-cooled gelated samples in PTFE moulds were mounted directly on the coldfinger of the freeze-caster, left open to ambient conditions of $\sim 5{ }^{\circ} \mathrm{C}$, equilibrated at a coldfinger temperature of 275 $\mathrm{K}$ for 600 seconds and frozen using a constant temperature change rate of $-1.5 \mathrm{~K} / \mathrm{min}$ until the sample was frozen solid.

Ice was removed from the frozen samples in a freeze-drier (Christ Alpha 1-2 LD plus, Buch \& Holm) for 24 hours. Green samples were then fired in air, initially burning out organic additives at $250{ }^{\circ} \mathrm{C}$ and $450{ }^{\circ} \mathrm{C}$ using a heating rate of $15 \mathrm{~K} / \mathrm{hour}$, followed by sintering at $1100{ }^{\circ} \mathrm{C}$ for 3 hours for samples with PVP and B-1022 with a heating rate of $30 \mathrm{~K} /$ hour. Given the evaluated onset temperatures in Table 1, a more cautious sintering temperature of $1000{ }^{\circ} \mathrm{C}$ with a heating rate of 30 $\mathrm{K} /$ hour was chosen for gelated samples, as these had a tendency to develop crack formations upon sintering at elevated temperatures.

\subsubsection{Structural \& chemical characterization}

The structural analysis of freeze-cast samples is based on image analysis of SEM micrographs and follows the procedure described by Christiansen et al.[6]. Here, samples are mounted in epoxy (EpoFix, resin and hardener, Struers), cut into smaller specimens revealing cross sections perpendicular and parallel to the freezing direction, polished and coated with a $\sim 12 \mathrm{~nm}$ thick carbon layer and imaged using a scanning electron microscope (SEM, TM3000, Hitachi HighTechnologies). Mean pore size of the ice-templated pores, macroporosity, i.e. the channel porosity, and specific surface area were evaluated from binarized SEM images obtained perpendicular to the freezing direction. Three images containing $\sim 300$ pores each were analyzed at each vertical sample position in order to obtain a statistically significant data set.
SEM micrographs can be found as supporting data (see section 5). The quantification of pore size and specific surface area from image analysis was originally developed for analysis of 3D data[19;20]. Structural parameters of pore size, macroporosity and specific surface area - the latter in this case determined as area per volume - are all determined from binarized images, thus removing structural features smaller than a few microns and thereby disregarding e.g. inter-particle porosity and the pore size of micropores in the walls of the freeze-cast structures.

The LCSM9-LCSM6/CGO interface was evaluated at cross sections parallel to the freezing direction by SEM micrographs and energy-dispersive x-ray spectroscopy (EDS, Bruker). EDS spectra for elemental analysis were collected at interfaces over $512 \times 384$ pixels (pixel size of $3.2 \times 3.2 \mu \mathrm{m}$, i.e. a total sample area of $1638 \times 1229 \mu \mathrm{m})$, with a $128 \mu \mathrm{s}$ dwell time per pixel and a total collection time of 600-1800 seconds. Accumulated EDS spectra either over a selection, as illustrated by yellow boxes in Figure 4, or over all pixels in a sample area were analyzed using Quantax Esprit 2.1 software (Bruker Microanalysis Software, Bruker), where deconvolution of peaks was achieved by series fit and quantified by standardless P/B-ZAF method.

The LCSM9-LCSM6/CGO interface was detected by evaluation of $\mathrm{Ce}$-content at a given sample position. Cerium has a distinct $\mathrm{L}_{\alpha}$ emission peak at $4.84 \mathrm{keV}$, as seen in the EDS spectra in Figure 4, where accumulated EDS spectra of the selection above and below the interface is shown on the right. The Ce-peak is only clearly visible for the selection below the interface, i.e. the LCSM6/CGO phase. The element analysis was carried out for signals from lanthanum, calcium, strontium, manganese and cerium, thus, the overall analysis yielded the relative $\mathrm{Ce}$-content of a given sample at a given position.

Accumulated, deconvoluted EDS spectra and identified background for all analysis points can be found as supporting data (see section 5).

\section{Results \& Discussion}

\subsection{Interface characterization}

Freeze-casting of LCSM suspensions with the implementation of stepwise, continuous, highly viscous and gelation freezing all resulted in a distinct layering of suspensions. This yielded a clearly visible and sharp interface between the two layers in both the green and sintered samples, as seen on Figure 5 The figure shows a picture of a green body freeze-cast sample, where the white CGO made the LCSM6/CGO phase grey and thereby visually identifiable.

Freeze-casting of a homogeneous suspension of particles generally results in structures with directional porosity in the form of microchannels along the freezing direction, which was also the case for samples in this study, as evident from Figure 6. However, what is remarkable, is that all procedures except for stepwise freezing, led to continuous channels across the interface. The interfaces in Figure 6 are enhanced 

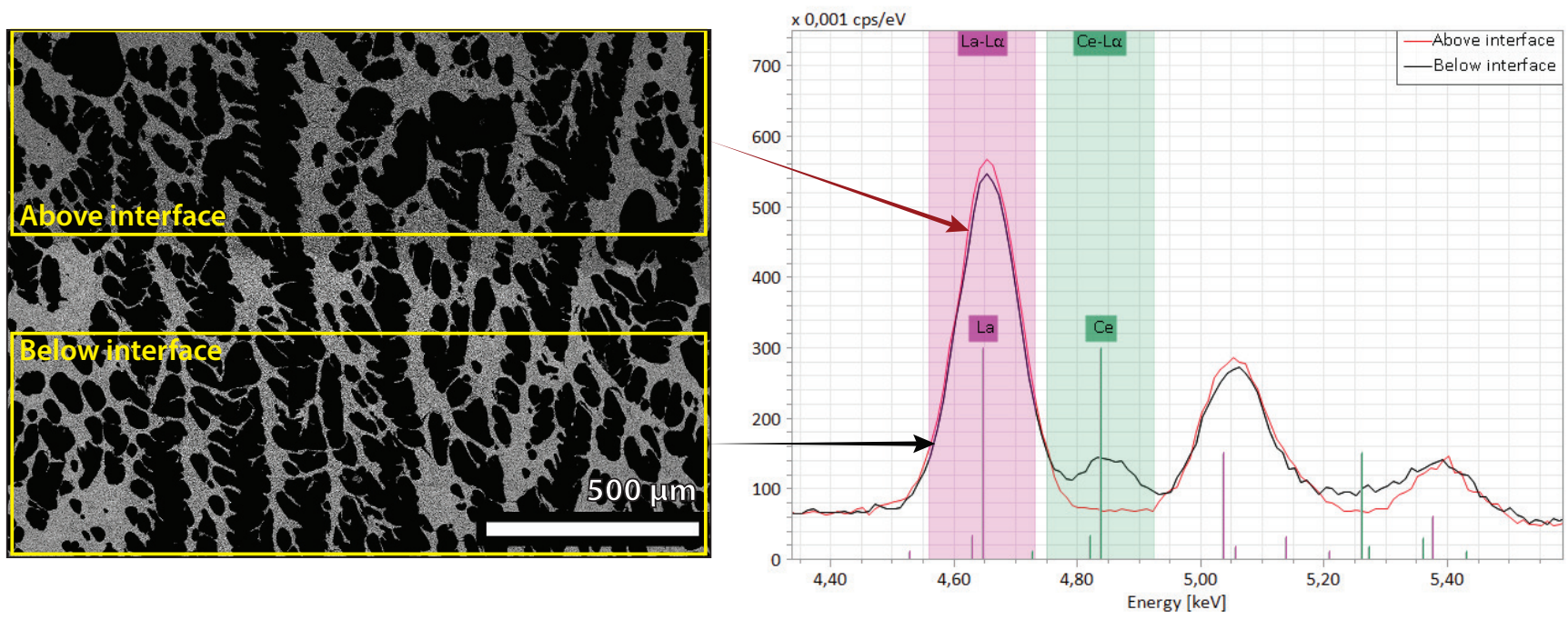

Figure 4. Accumulated EDS spectra obtained above and below the LCSM9 and LCSM6/CGO interface, respectively, of a gelated freeze-cast sample. The analyzed region of the sample is indicated by the yellow boxes. The Cerium $\mathrm{L}_{\alpha}$-peak at $4.84 \mathrm{keV}$ is only clearly distinguishable in the accumulated spectra of the selection below the interface.

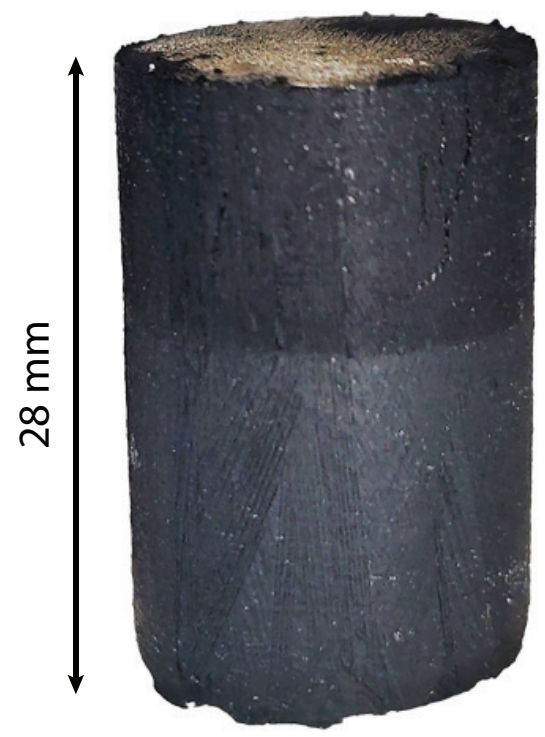

Figure 5. Picture of freeze-cast green sample frozen from LCSM6/CGO (grey bottom layer) and LCSM9 (black top layer) suspensions with 5 wt\% PVP K30.

by adjusting the grayscale threshold of SEM images and correlating these with Ce-mapping in EDS analytical software. For ease of visualization in compressed and printed images a semi-transparent dashed line indicating the interface has been added as an eye-guide.

The relative $\mathrm{Ce}$-content above and below the interfaces given in Figure 6 are evaluated as described in Figure 4. However, standardless EDS analysis is not sufficient for an exact quantification of the elemental composition. For comparison, EDS elemental analysis of a freeze-cast sample of only LCSM6 still yields a relative atomic Ce-content of $0.32 \%$.
Although, as the qualitative elemental composition of samples are known, the measured relative Ce-contents can still be evaluated between samples and are in this work thus used as a both visual and chemical marker of the LCSM6 phase.

A significant discontinuity in channels across the interface for the stepwise frozen sample is seen in Figure 6a), with evidently altered channel geometry in the form of more narrow channels above the interface. The channel geometry in freeze-cast ceramics is directly shaped from the growing ice crystals during the freezing phase. The growth of ice crystals in ceramic suspensions during freeze-casting has previously been observed by in situ X-ray[21; 22]. Upon freezing, rapid nucleation takes place at the cold side forming ice crystals that grow in random directions. This is the isotropic region with no resulting directional porosity. Eventually, ice crystals growing along the direction of the temperature gradient are favoured, resulting in a region of aligned crystals. This region is referred to as the steady state region or the anisotropic region. When freezing samples stepwise, the initial suspension frozen in the first step act as a cold side for the second suspension and a new nucleation zone occurs at the interface. This results in a second isotropic region and thus discontinuous channels as is evident from the significant size difference of channels above and below the dashed line in Figure 6a). To avoid a second nucleation zone, the second suspension was added before the freezing front had reached the top of the suspension, i.e. while the top of the first suspension was still liquid, and samples where then frozen continuously, eliminating the second isotropic region as seen on Figure 6b) and ensuring continuous channels.

Generally, freezing conditions have been chosen such that they could be kept consistent and reproducible, however, the continuous freezing conditions are much more difficult to reproduce as they rely on exact determination of the freez- 
(a) Stepwise freezing

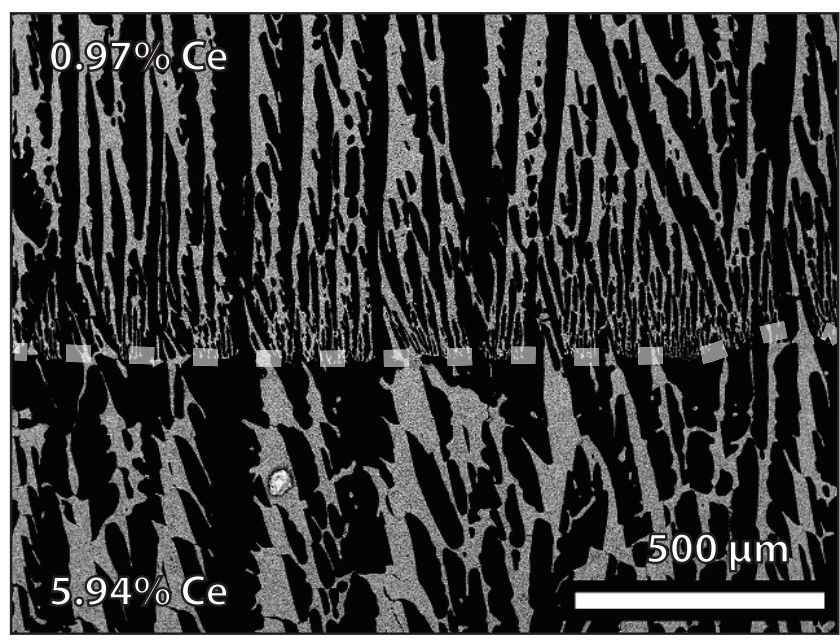

(c) Viscocity

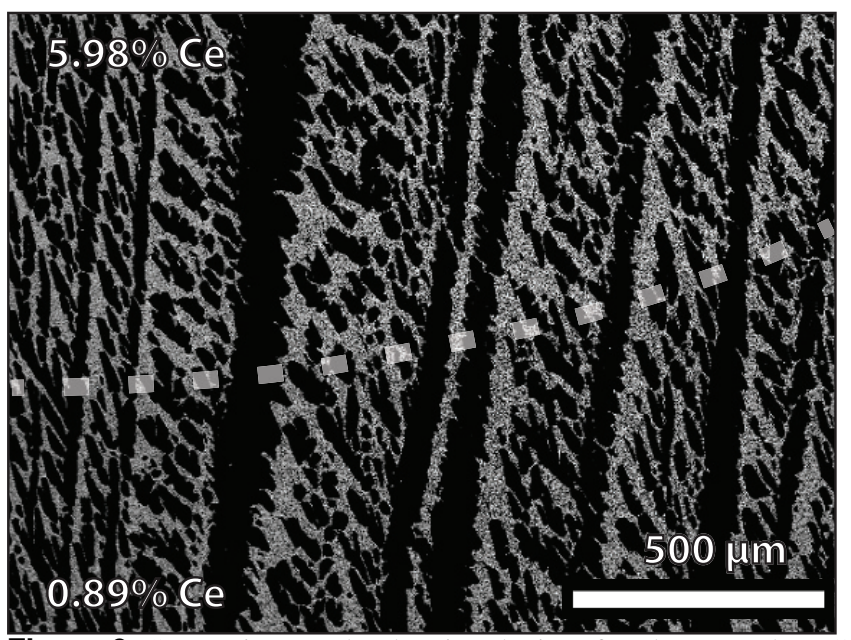

(b) Continuous freezing

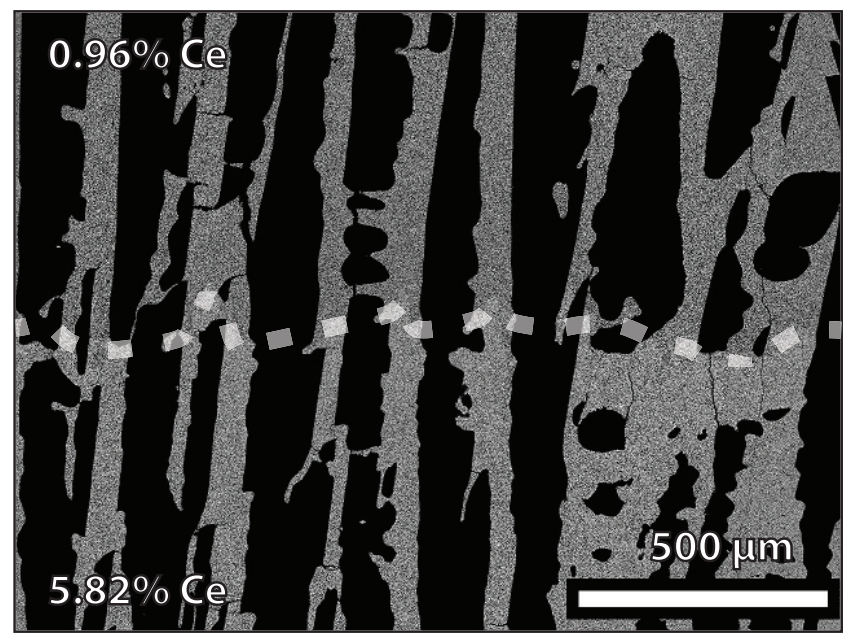

(d) Gelation freezing

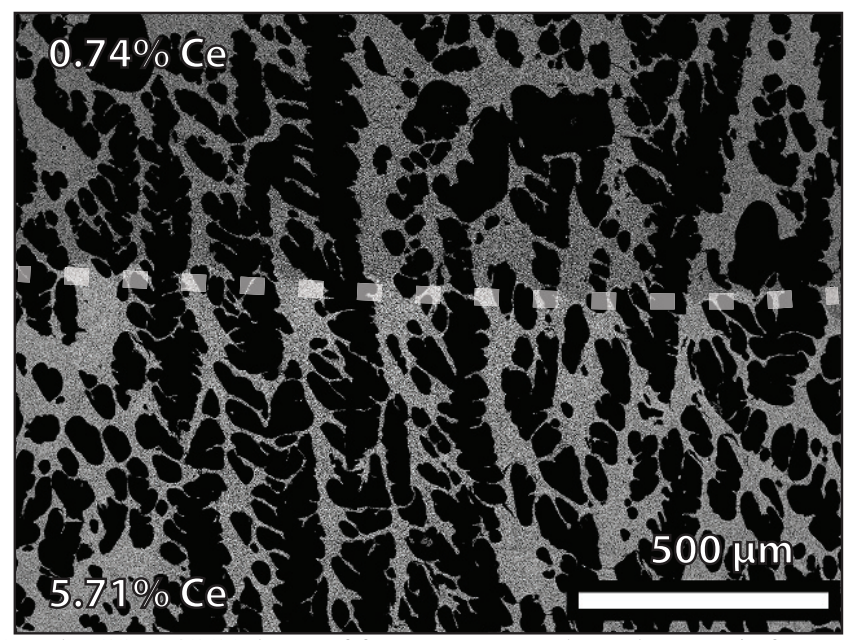

Figure 6. SEM micrographs showing the interface between the LCSM9 and LCSM9/CGO layer of freeze-cast samples, where (a) is frozen stepwise, (b) is frozen continuously, (c) is frozen from a suspension with 10\% PVP K30 for increased viscosity, and (e) is gelated prior to being frozen. The measured relative atomic content of Cerium for the two layers are indicated in the left corners and the dashed lines are eye-guides to emphasize the interface between the two layers. Images are obtained at cross sections parallel to the freezing direction at the approximate center of the samples. Note that (a-b) and (d) are frozen with LCSM6/CGO layer at the bottom, while (c) is frozen with the LCSM6/CGO layer at the top.

ing front position. Given that the spatial resolution of thermocouples is $4 \mathrm{~mm}$ in our freeze-casting set-up[18], there will always be an uncertainty in immediate determination of freezing-front position and thus inconsistent freezing conditions. Additionally, the freezing front position is estimated at the thermocouple where the temperature is $0{ }^{\circ} \mathrm{C}$, however, freezing point depression due to organic additives and ceramic particulates will lower the actual freezing point of the suspension and thereby increase the uncertainty of the freezing front position. By extension, it should be mentioned, that continuously frozen samples for which the position of the freezing front was underestimated during the continuous freezing resulted in discontinuous channels across the interface similar to those of stepwise freezing, as the conditions for these samples more closely resembled the latter procedure. However, as the interface does appear sharp and more or less planar with continuous channels across, continuous freezing of suspensions is a feasible processing route for achieving distinct layers in a freeze-cast structure for freeze-casting set-ups with precise and immediate monitoring of the freezing front. An obvious approach for immediate tracking of the freezing front is visually. As the freezing front has been found to be a well-defined, slightly concave planer interface perpendicular to the freezing direction[22], visual tracking has been successful in previous freeze-casting studies with the application of an acrylic glass mould equipped with a scale bar[5; 4]. However, due to the color of LCSM, the freezing front cannot be tracked visually[23]. 


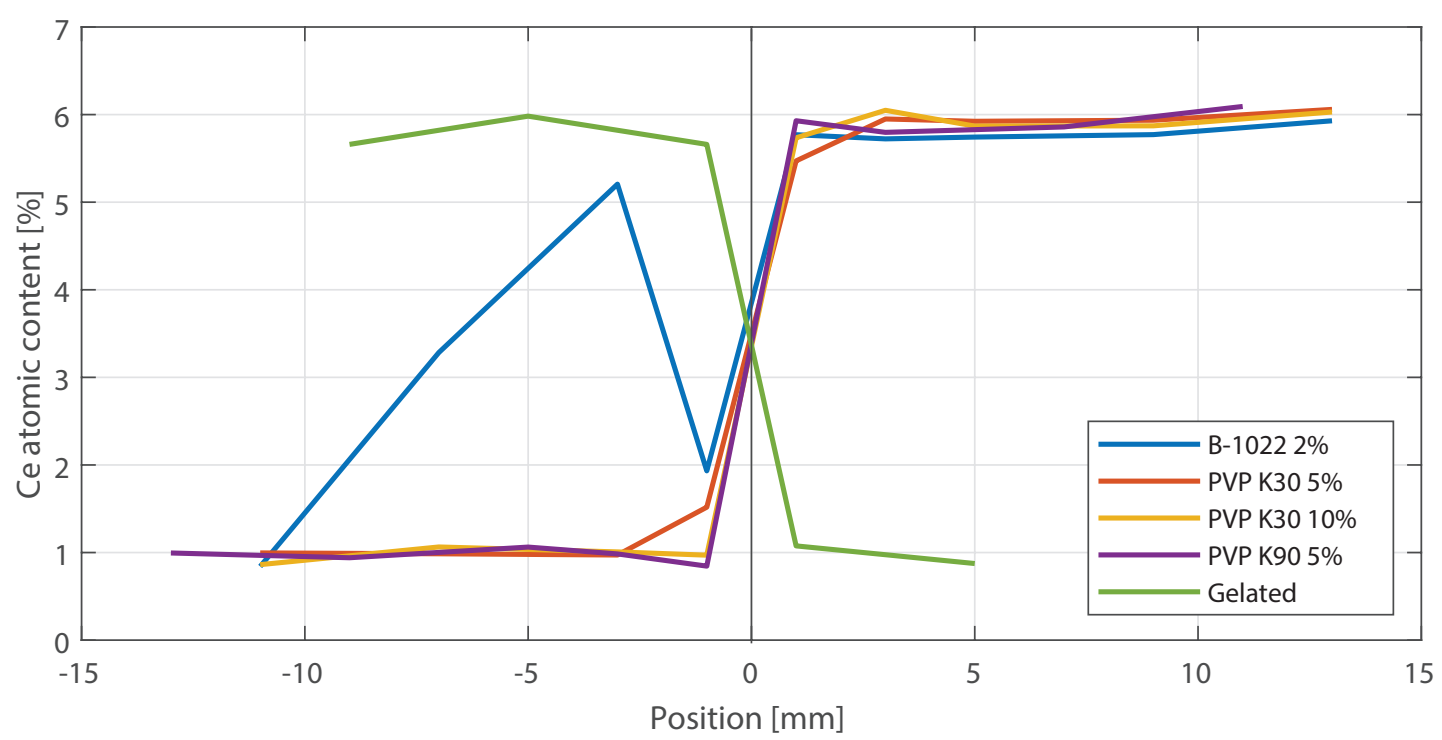

Figure 7. Relative atomic Ce-content as a function of sample height for samples with varying viscosity or an initial gelation-step prior to freezing, evaluated using EDS elemental analysis. The greater the viscosity, the sharper the interface. Note that samples with B-1022 binder and PVP polymers are frozen with the LCSM6/CGO layer at the top, while the gelated sample is frozen with the LCSM6/CGO layer at the bottom.

\subsection{Structural continuity across interfaces}

As the freezing conditions for continuously frozen samples could not be kept consistent and stepwise frozen samples showed a clear discontinuity in channels across the LCSM9-LCSM6/CGO interface, a structural analysis was only carried out for freeze-cast samples frozen from viscous or gelated suspensions. Both of these displayed continuous channels across a distinct interface as is clear from Figure $6 c-d)$.

Various viscosities of suspensions were tested in order to clarify the effect of viscosity on the sharpness and possibly the shape of the LCSM9-LCSM6/CGO interface. With the addition of long polymer chains in the form of PVP, the intermolecular forces and entanglement between polymer chains resist flow and thus miscibility of suspensions. The effect increases with increased concentration and length of polymers, e.g. the PVP K90 consists of on average longer chains than PVP K30 and thus results in greater viscosity at similar concentrations. In Figure 6c) the interface of a freeze-cast frozen from highly viscous suspensions with $10 \mathrm{wt} \%$ PVP K30 is shown. The interface is rather concave in shape. This shape is in accordance with the sample preparation procedure where the LCSM6/CGO suspension is added at the center, pushing the other suspension radially aside, which will inevitably create a concave interface provided that the two suspensions do not mix.

The sharpness and position of LCSM9-LCSM6/CGO interfaces are evaluated by analyzing the relative Ce-content as a function of sample height in the center of the samples as in Figure 7, where the sample position is normalized at the interface. An increase in relative $\mathrm{Ce}$-content thus indicates a transition from one layer to the other, where the steeper the curve the sharper the interface. The miscibility of viscous solutions appear to generally be very low and all interfaces are sharp with a steep increase in relative Ce-content. All samples follow this trend, except for the sample frozen from suspensions with the commercial B-1022 binder and thus low viscosity. Here, a significant peak in relative Ce-content is found below the interface, followed by a drop in Ce-content immediately before a steep increase indicating the interface beyond which the relative Ce-content is more or less constant. This is due to the miscibility of the two suspensions, where, presumably, a drop of the LCSM6/CGO suspension has sunk into the LCSM9 phase and mixed. Additionally, the interface of the samples frozen from suspensions with only $5 \mathrm{wt} \% \mathrm{PVP}$ $\mathrm{K} 30$ and thus the second lowest viscosity appears less sharp than for samples frozen from suspension of higher viscosity as the increase in relative Ce-content stretches over $4 \mathrm{~mm}$, indicating a more blurred interface. Although a sharp interface is not achieved, these results hint that a graded interface could possibly be achieved by application of suspensions of fine-tuned viscosities somewhere between that of the B-1022 suspensions and that of the $5 \mathrm{wt} \%$ PVP K30 suspensions. Alternatively, a graded interface could be achieved by layering of three or more highly viscous suspensions with one or more mixed LCSM6/LCSM9 phase(s) between the two pure ones.

The freezing kinetics and thus resulting channel size and shape strongly depend on suspension properties such as particle size, viscosity and powder load[3; 24]. Extra care was therefore taken to ensure equivalent particle loads, viscosities and particle sizes of both the LCSM9 and LCSM6/CGO suspension, as described in section 2.1, which ensured structural 
continuity across the interface. Figure 8a-c) show structural properties of samples frozen from viscous and gelated suspensions. Pore size, porosity and specific surface area in Figure 8 are those due to the ice-templated pores, as it is the structural homogeneity of these that are investigated. Continuity of all structural features is maintained across the interface, however, with a slightly increasing mean pore size for all samples in Figure 8a). Although, it is not unambiguous that the increase in mean pore size is due to changes in suspension properties. A direct indicator of the freezing kinetics is the estimated freezing front velocities as shown in Figure 8d), where a slight decrease in freezing front velocities moving from the bottom of samples across the interface to the top of samples is seen. As the pore size in freeze-cast ceramics is directly related to the freezing front velocity $[3 ; 4 ; 5]$ the slight increase in pore sizes probably arises from the slight decrease in freezing front velocities, as the freezing front velocities were not controlled directly in this work. Only the freezing rate was controlled and kept constant, as all samples were frozen using a linear freezing profile where the temperature of the coldfinger was decreased at $-1.5 \mathrm{~K} / \mathrm{min}$, resulting in a freezing front velocity of $\sim 13 \mu \mathrm{m} / \mathrm{s}$.

The same trends are also observed for the gelated sample in Figure 8a-c); i.e. structural continuity is maintained across the LCSM6/CGO-LCSM9 interface. For gelated samples the interface between the two layers of LCSM9 and LCSM6/CGO directly reflects the shape of the cut-out specimens of gelated suspensions and thus gelation of suspensions prior to freezing ensures a sharp and planar interface, as seen on Figure 6d). With a homogeneous and proper gelation, the shape - or possible tilt, if so desired - of the interface can be fine-tuned along with the layer thickness and geometry. However, introducing a gelation-step also introduces additional possibilities of structural failure, as shown in Figure 9. Figure 9a) illustrates delamination which can occur if the stacking of gelated suspensions are sloppy. Samples that were not stacked carefully prior to freezing would often delaminate and fracture along the interface.

Another critical failure in gelated freeze-cast structures is due to circular voids in the fired structure due to bubbles in the suspension, as can be seen on Figure 9b). As the suspension is heated and stirred using a propeller immediately before gelation, it is possible that air will be stirred into the solution forming bubbles. The freeze-cast in Figure 9b) was left to gelate without being de-aired in vacuum, and thus, circular cavities were found in the fired ceramic. De-airing the suspension immediately after it had been transferred to the mould proved highly efficient for eliminating bubbles and thus cavities.

\subsection{Structural geometry and additives}

Changing the suspension composition and properties significantly changes the resulting pore morphology as revealed in Figure 8a-c) where changes of the pore, or channel, morphology due to the addition of either PVP or gelation freezing is

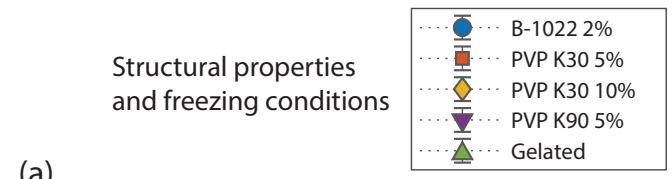

(a)

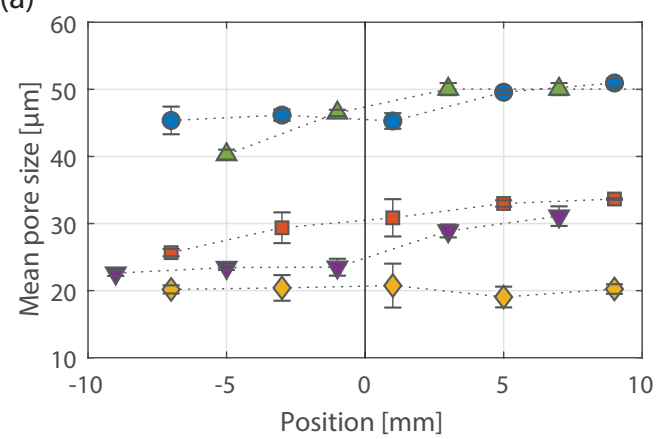

(b)

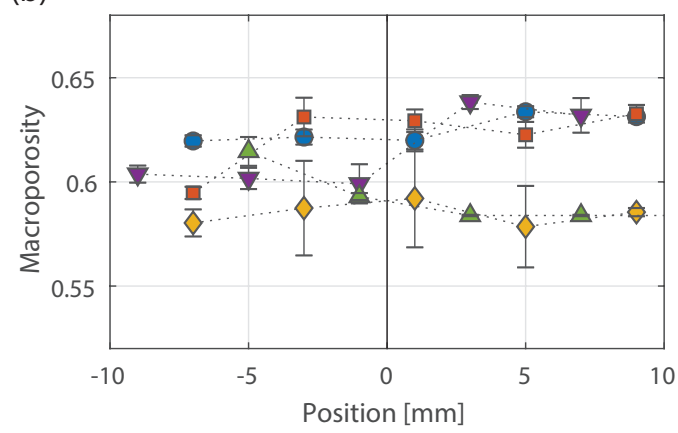

(c)

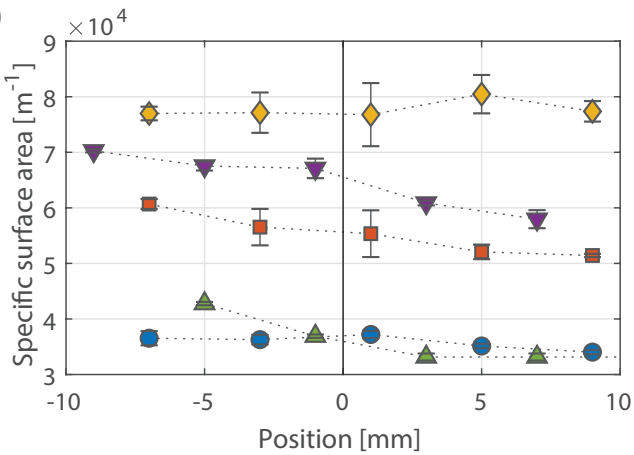

(d)

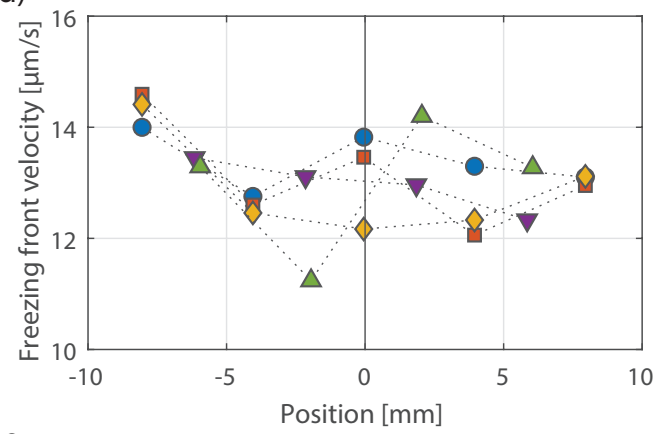

Figure 8. Structural characterization of freeze-cast samples across the LCSM6/CGO - LCSM9 interface, where (a) mean pore size, (b) macroporosity and (c) specific surface area are all evaluated from image analysis of SEM micrographs. In (d) estimated freezing front velocities throughout the samples during freezing are given. All samples are frozen using the same constant freezing rate of the coldfinger of $-1.5 \mathrm{~K} / \mathrm{min}$. 


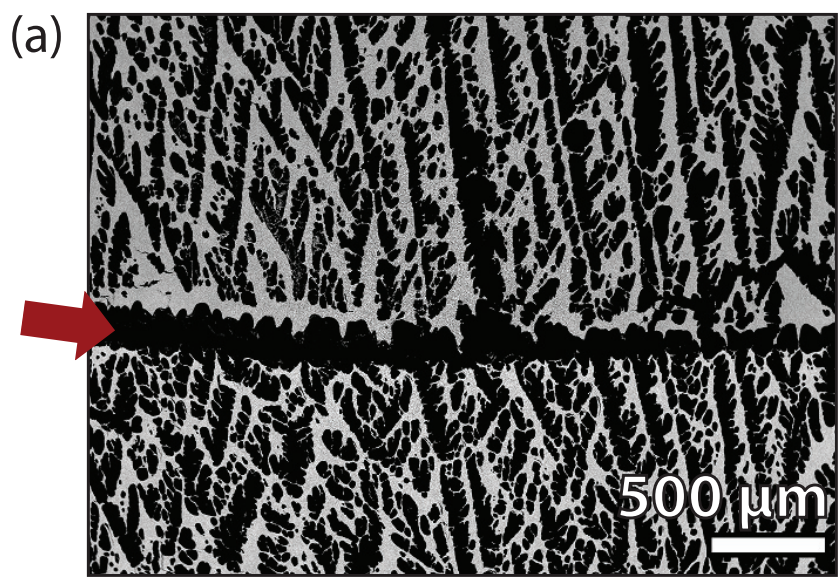

(b)

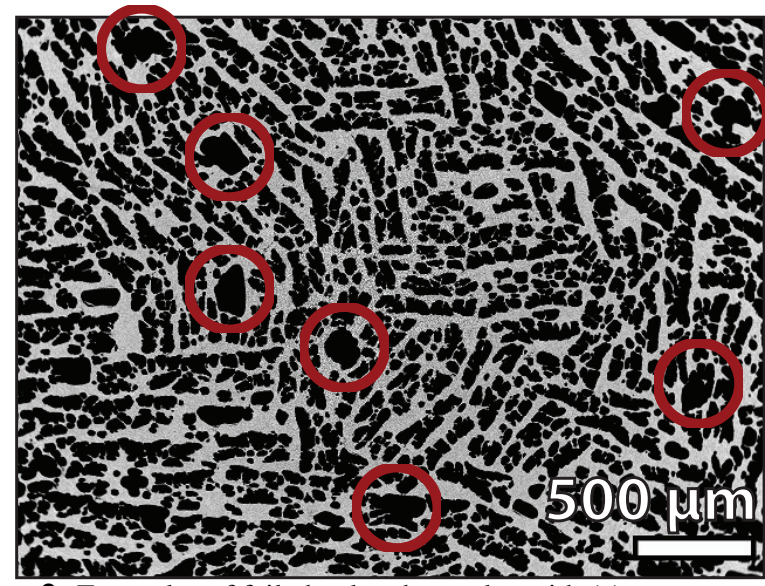

Figure 9. Examples of failed gelated samples with (a) delamination along the interface due to faulty stacking of gelated specimens and (b) circular cavities in the structure due to bubbles in the suspension that was trapped during gelation.

evident from the changing structural features.

While the measured macroporosity is more or less the same for all samples independent of additives, Figure 8b), this is not the case for pore sizes, Figure 8a). Pore sizes of freeze-casts prepared from highly viscous suspensions generally exhibit smaller pore sizes than samples frozen from low viscosity or gelated suspensions. During freezing, the long, entangled polymer chains presumably inhibit the fluidity of the highly viscous suspensions, thereby limiting the diffusion of water molecules to the growing ice crystals, resulting in smaller ice crystals and thereby smaller pores in the fired freeze-cast. Accordingly, the inverse phenomena is seen for the specific surface area, Figure 8d), which is larger with increased viscosity and thus smaller pore sizes. The addition of PVP and pre-gelation does not seem to significantly affect the estimated freezing front velocities. Ice crystal growth in the $\mathrm{z}$-direction, i.e. along the thermal gradient, during freezecasting is thus not significantly affected by PVP or gelatin, meanwhile, the radial growth of ice crystals is suppressed due to limited diffusion of water.

A more thorough discussion of the effect on the pore morphology due to addition of polymers resulting in an increased viscosity $[24]$ and pre-gelation using gelatine $[9 ; 10 ; 6]$ has previously been studied and is thus beyond the scope of this work.

\section{Conclusion}

Functionally graded freeze-casts of LCSM6 and LCSM9 with continuous microchannels and structural integrity were achieved.

Freeze-casting of LCSM suspensions with the implementation of stepwise, continuous, highly viscous and gelation freezing all resulted in a distinct layering of suspensions. This yielded a clearly visible and sharp interface between the two layers in both the green and sintered samples. The latter three procedures resulted in continuous channels across the interface.

The two chemically very similar phases of LCSM6 and LCSM9 were successfully distinguished from one another by the addition of $10 \mathrm{wt} \%$ CGO to the LCSM6 phase, making it possible to track this phase using EDS analysis in order to evaluate the relative atomic Ce-content and using this as a marker.

Keeping the solid load and particle size distribution of all suspensions consistent, while ensuring an approximate constant freezing front velocity of $\sim 13 \mu \mathrm{m} / \mathrm{s}$ by implementing a constant temperature change rate of $-1.5 \mathrm{~K} / \mathrm{min}$ during freeze-casting, a structural continuity was maintained across the LCSM9-LCSM6/CGO interface. While the interface was concave in shape for frozen viscous suspensions, the interface of gelated samples directly reflected the shape of the cut-out specimens of gelated suspensions, thus ensuring a sharp and planar interface.

The overall pore morphology was found to reflect the changes in suspension properties. While the macroporosity of freeze-casts was found to be independent of changes in additives and gelation, the overall pore size of freeze-casts was found to decrease with an increase in PVP concentration and thus the viscosity. Both gelation and addition of PVP changes the pore morphology of freeze-cast specimens.

\section{Supporting information \& data}

Supporting information and supporting data in the form of SEM micrographs and EDS spectra are available at: 10.11583/DTU.9878894

\section{Acknowledgements}

This work is funded by the Independent Research Fund Denmark - Technologies and Productions Sciences, project no. 6111-00073B. C. D. Christiansen moreover wishes to acknowledge the contributions of Karl T. S. Thydén, Jacob R. Bowen, Ebtisam Abdellahi, Lene Knudsen and Christian R. H. Bahl, Technical University of Denmark, in the form of valuable discussions and insights. 


\section{References}

[1] K. L. Scotti, D. C. Dunand, Freeze casting - A review of processing, microstructure and properties via the open data repository, FreezeCasting.net, Progress in Materials Science 94 (2018) 243-305. doi:10.1016/j. pmatsci.2018.01.001.

[2] S. Deville, E. Saiz, R. K. Nalla, A. P. Tomsia, Freezing as a path to build complex composites., Science 311 (5760) (2006) 515-518. doi:10.1126/ science. 1120937.

[3] S. Deville, E. Saiz, A. P. Tomsia, Ice-templated porous alumina structures, Acta Materialia 55 (6) (2007) 19651974. doi:10.1016/j.actamat.2006.11.003.

[4] T. Waschkies, R. Oberacker, M. J. Hoffmann, Control of lamellae spacing during freeze casting of ceramics using double-side cooling as a novel processing route, Journal of the American Ceramic Society 92 (2009) 79-84. doi : $10.1111 / j .1551-2916.2008 .02673 . x$.

[5] A. Z. Lichtner, D. Jauffrès, C. L. Martin, R. K. Bordia, Processing of hierarchical and anisotropic porosity LSMYSZ composites, Journal of the American Ceramic Society 96 (9) (2013) 2745-2753. doi: $10.1111 /$ jace. 12478.

[6] C. D. Christiansen, K. K. Nielsen, R. K. Bordia, R. Bjørk, The effect of gelation on statically and dynamically freeze-cast structures, Journal of the American Ceramic Society 102 (2019) 5796-5806. doi : 10.1111 / jace. 16500.

[7] M. Acosta, V. L. Wiesner, C. J. Martinez, R. W. Trice, J. P. Youngblood, Effect of polyvinylpyrrolidone additions on the rheology of aqueous, highly loaded alumina suspensions, Journal of the American Ceramic Society 96 (5) (2013) 1372-1382. doi:10.1111/jace.12277.

[8] D. Marani, B. R. Sudireddy, W.-R. Kiebach, L. Nielsen, S. Ndoni, Rheological properties of poly(vinylpiyrrolidone) as a function of molecular weight, Nordic Rheology Society.Annual Transactions 22 (2014).

[9] M. Fukushima, Y. I. Yoshizawa, T. Ohji, Macroporous ceramics by gelation-freezing route using gelatin, Advanced Engineering Materials 16 (6) (2014) 607-620. doi:10.1002/adem.201400067.

[10] M. Fukushima, T. Ohji, H. Hyuga, C. Matsunaga, Y. I. Yoshizawa, Effect of gelatin gel strength on microstructures and mechanical properties of cellular ceramics created by gelation freezing route, Journal of Materials Research 32 (17) (2017) 3286-3293. doi:10.1557/ jmr. 2017.94 .
[11] A. Dinesen, S. Linderoth, S. Mørup, Direct and indirect measurement of the magnetocaloric effect in La 0.67 Ca 0.33-x Sr x MnO 3, Journal of Physics: Condensed Matter 17 (39) (2005) 6257-6269. doi:10.1088/ 0953-8984/17/39/011.

[12] T. Lei, K. Engelbrecht, K. K. Nielsen, C. T. Veje, Study of geometries of active magnetic regenerators for room temperature magnetocaloric refrigeration, Applied Thermal Engineering 111 (2017) 1232-1243. doi:10.1016/ j.applthermaleng.2015.11.113.

[13] C. R. H. Bahl, D. Velzáquez, K. K. Nielsen, K. Engelbrecht, K. B. Andersen, R. Bulatova, N. Pryds, High performance magnetocaloric perovskites for magnetic refrigeration, Applied Physics Letters 100 (12) (2012). doi:10.1063/1.3695338.

[14] A. Smith, C. Bahl, R. Bjørk, K. Engelbrecht, K. K. Nielsen, N. Pryds, Materials challenges for high performance magnetocaloric refrigeration devices, Advanced Energy Materials 2 (11) (2012) 1288-1318. doi : 10 . 1002 / aenm. 201200167.

[15] J. Liang, C. D. Christiansen, K. Engelbrecht, K. K. Nielsen, R. Bjørk, C. R. Bahl, Heat Transfer and Flow Resistance Analysis of a Novel Freeze-Cast Regenerator, (submitted).

[16] P. Govindappa, P. V. Trevizoli, O. Campbell, I. Niknia, T. V. Christiaanse, R. Teyber, S. Misra, M. A. Schwind, D. Van Asten, L. Zhang, A. Rowe, Experimental investigation of mnfep1-xasx multilayer active magnetic regenerators, Journal of Physics D: Applied Physics 50 (31) (2017) 315001. doi : 10 . 1088/1361-6463/ aa7a33.

[17] R. Bulatova, C. Bahl, K. Andersen, L. T. Kuhn, N. Pryds, Functionally Graded Ceramics Fabricated with Side-bySide Tape Casting for Use in Magnetic Refrigeration, International Journal of Applied Ceramic Technology 12 (4) (2015) 891-898. doi:10.1111/ijac.12298.

[18] C. D. Christiansen, K. K. Nielsen, R. Bjørk, Novel freezecasting device with high precision thermoelectric temperature control for dynamic freezing rates, (submitted) (2020).

[19] P. Jørgensen, S. Ebbehøj, A. Hauch, Triple phase boundary specific pathway analysis for quantitative characterization of solid oxide cell electrode microstructure, Journal of Power Sources 279 (2015) 686-693. doi : $10.1016 / j$.jpowsour.2015.01.054.

[20] S. De Angelis, P. Jørgensen, E. Tsai, M. Holler, K. Kreka, J. Bowen, Three dimensional characterization of nickel coarsening in solid oxide cells via ex-situ ptychographic nano-tomography, Journal of Power Sources 383 
(2018) 72-79. doi:10.1016/j.jpowsour.2018. 02.031.

[21] S. Deville, E. Maire, A. Lasalle, A. Bogner, C. Gauthier, J. Leloup, C. Guizard, In Situ X-ray radiography and tomography observations of the solidification of aqueous alumina particles suspensions. Part II: Steady state, Journal of the American Ceramic Society 92 (11) (2009) 2497-2503. doi:10.1111/j.1551-2916.2009. $03264 . x$.

[22] A. Bareggi, E. Maire, A. Lasalle, S. Deville, Dynamics of the freezing front during the solidification of a colloidal alumina aqueous suspension: In situ x-ray radiography, tomography, and modeling, Journal of the American Ceramic Society 94 (10) (2011) 3570-3578. doi:10.1111/j.1551-2916.2011.04572.x.

[23] C. D. Christiansen, K. K. Nielsen, R. Bjørk, Freezecasting to create create directional micro-channels in regenerators for magnetic refrigeration, in: Proceedings of TherMag VIII, 8th IIF-IIR International Conference on Caloric Cooling, Darmstadt, Germany, 2018, pp. 96-101. doi:10.18462/iir.11072.

[24] M. M. Porter, R. Imperio, M. Wen, M. A. Meyers, J. McKittrick, Bioinspired scaffolds with varying pore architectures and mechanical properties, Advanced Functional Materials 24 (14) (2014) 1978-1987. doi: 10. 1002 /adfm.201302958. 